Research Article

\title{
Global Dynamics, Bifurcation Analysis, and Chaos in a Discrete Kolmogorov Model with Piecewise-Constant Argument
}

\author{
Abdul Qadeer Khan (iD) \\ Department of Mathematics, University of Azad Jammu and Kashmir, Muzaffarabad 13100, Pakistan \\ Correspondence should be addressed to Abdul Qadeer Khan; abdulqadeerkhan1@gmail.com
}

Received 26 July 2021; Revised 7 September 2021; Accepted 22 September 2021; Published 5 November 2021

Academic Editor: M Syed Ali

Copyright (c) 2021 Abdul Qadeer Khan. This is an open access article distributed under the Creative Commons Attribution License, which permits unrestricted use, distribution, and reproduction in any medium, provided the original work is properly cited.

\begin{abstract}
The local behavior with topological classifications, bifurcation analysis, chaos control, boundedness, and global attractivity of the discrete-time Kolmogorov model with piecewise-constant argument are investigated. It is explored that Kolmogorov model has trivial and two semitrival fixed points for all involved parameters, but it has an interior fixed point under definite parametric condition. Then, by linear stability theory, local dynamics with different topological classifications are investigated around trivial, semitrival, and interior fixed points. Further for the discrete Kolmogorov model, existence of periodic points is also investigated. It is also investigated the occurrence of bifurcations at interior fixed point and proved that at interior fixed point, there exists no bifurcation, except flip bifurcation by bifurcation theory. Next, feedback control method is utilized to stabilize chaos existing in discrete Kolmogorov model. Boundedness and global attractivity of the discrete Kolmogorov model are also investigated. Finally, obtained results are numerically verified.
\end{abstract}

\section{Introduction}

1.1. Motivation and Literature Review. There are many symbiotic interactions existing in nature between two or more species in an ecosystem. Mutualism is an example of such interaction where interacted species get benefit from each other. For example, termites eat cellulose of wood but cannot digest it, and flagellates reside in the termite's gut decomposing the cellulose of food and thus providing nutrients to termites. On the other hand, termite's gut provides food and shelter to flagellates. It is pointed out that in theoretical ecology, mutualist behavior of symbiosis or mutualism is very significant [1]. This field is not widely studied as the other fields of mathematical biology even for two species, although its importance is equal to the other competitive interactions such as host-parasitoid and preypredator interactions. So, this topic of mutualism system seems interesting to study. For instance, May [2] suggested the two-species hybrid continuous-time Kolmogorov model represented by the following system of differential equations:

$$
\begin{aligned}
& \frac{\mathrm{d} x}{\mathrm{~d} t}=r_{1} x(t)\left(1-\frac{x(t)}{\beta_{1}+\alpha_{1} y([t])}\right), \\
& \frac{\mathrm{d} y}{\mathrm{~d} t}=r_{2} y(t)\left(1-\frac{y(t)}{\beta_{2}+\alpha_{2} x([t])}\right),
\end{aligned}
$$

where $[t]$ represents the greatest integer in $t$, and parameters $r_{1}, r_{2}, \alpha_{1}, \alpha_{2}, \beta_{1}$, and $\beta_{2}$ are positive numbers. Moreover, due to the effect of piecewise-constant argument, we have integrated both sides of the Kolmogorov model, which is depicted in (1). On interval $t \in[n, n+1)$, (1) can be written as

$$
\begin{aligned}
& \frac{\mathrm{d} x(t)}{\mathrm{d} t}-r_{1} x(t)=-\frac{r_{1}(x(t))^{2}}{\beta_{1}+\alpha_{1} y(n)}, \\
& \frac{\mathrm{d} y(t)}{\mathrm{d} t}-r_{2} y(t)=-\frac{r_{2}(y(t))^{2}}{\beta_{2}+\alpha_{2} x(n)},
\end{aligned}
$$

where for $n=0,1, \ldots$, and $t \longrightarrow n+1$ resulted in the following solution: 


$$
\begin{aligned}
& x_{n+1}=\frac{e^{r_{1}} x_{n}}{1+\left(\left(e^{r_{1}}-1\right) /\left(\beta_{1}+\alpha_{1} y_{n}\right)\right) x_{n}}, \\
& y_{n+1}=\frac{e^{r_{2}} y_{n}}{1+\left(\left(e^{r_{2}}-1\right) /\left(\beta_{2}+\alpha_{2} x_{n}\right)\right) y_{n}} .
\end{aligned}
$$

It is important here to mention that discrete-time models directed by difference equations are more appropriate than the continuous ones in the case where populations have nonoverlapping generations, and also these models provide efficient computational results as compared to continuous models. Due to that reason in recent years, many scholars, researchers, and scientists have studied the dynamics of biological systems such as ratio-dependent predator-prey system, discrete hyperchaotic system, and host-parasitoid model. For instance, Bhattacharya and Saha [3] studied the dynamics characteristics of discrete Kolmogorov system. Cheng and Cao [4] explored dynamic characteristics of a discrete ratio-dependent prey-predator model. Jing and Yang [5] explored the dynamic characteristics of discrete prey-predator model. Kangalgil and Topsakal [6] explored dynamics characteristics of a discrete prey-predator system. Ran et al. [7] explored the Neimark-Sacker bifurcation of a stochastic discrete hyperchaotic system. Beddington et al. [8] explored dynamic characteristics in prey-predator models. Chen [9] studied the global dynamics and permanence of a discrete multispecies system. Lu and Zhang [10] investigated global attractivity and permanence of a discrete system with Holling type-II functional response. Fang and Chen [11] studied permanence of a discrete multispecies Lotka-Volterra model with delays. Fang et al. [12] investigated the dynamic characteristics of a discrete system. Jana and Samanta [13] studied prey-predator system in discrete-time scale using interval parameters.

1.2. Objective, Contributions, and Novelties. Motivated from aforementioned studies, the objective of the present work is to explore the global dynamics, bifurcations, and chaos in a discrete Kolmogorov model with piecewise-constant argument (3). More precisely, our main finding in this paper includes

Topological classifications at fixed points of the discrete Kolmogorov model (3) by linear stability theory

Exploration of periodic points of the discrete Kolmogorov model (3)

Flip bifurcation analysis at interior fixed point by bifurcation theory.

Investigation of chaos by feedback control method

To explore boundedness and global attractivity of discrete Kolmogorov model

Validation of obtained results numerically

1.3. Paper Structure. The rest of the paper is structured as follows: Section 2 relates with the investigation of topological classifications of discrete Kolmogorov model (3) at fixed points. In Section 3, periodic points of prime period-1 and period $-2,3, \ldots, n$ of the discrete Kolmogorov model (3) are explored, whereas comprehensive analysis of the bifurcation at fixed point $S$ is explored in Section 4. In Section 5, chaos control is explored by feedback control method, whereas Section 6 is about the presentation of numerical simulations to validate obtained results. In Section 7, boundedness and global dynamics are explored. The conclusion and future work are given in Section 8.

\section{Topological Classifications of Discrete Kolmogorov Model (3) at Fixed Points}

Here, local dynamical properties with topological classifications at fixed points are explored in $\mathbb{R}_{+}^{2}=\{(x, y): x, y \geq 0\}$. For this, first it is easy to verify that $\forall r_{1}, r_{2}, \alpha_{1}, \alpha_{2}, \beta_{1}, \beta_{2}$ discrete Kolmogorov model (3) has trivial fixed point $P=(0,0)$, boundary fixed points $Q=\left(0, \beta_{2}\right)$, and $R=\left(\beta_{1}, 0\right)$, but if $\alpha_{1}<\left(1 / \alpha_{2}\right)$, then it has an interior fixed point $S=\left(\left(\left(\beta_{1}+\alpha_{1} \beta_{2}\right) /\left(1-\alpha_{1} \alpha_{2}\right)\right),\left(\left(\beta_{2}\right.\right.\right.$ $\left.\left.\left.+\alpha_{2} \beta_{1}\right) /\left(1-\alpha_{1} \alpha_{2}\right)\right)\right)$. Additionally, the variational matrix $V$ at fixed point $(x, y)$ under the following map,

$$
\left(f_{1}, f_{2}\right) \mapsto\left(x_{n+1}, y_{n+1}\right) \text {, }
$$

where

$$
\begin{aligned}
& f_{1}=\frac{e^{r_{1}} x\left(\beta_{1}+\alpha_{1} y\right)}{\beta_{1}+\alpha_{1} y+\left(e^{r_{1}}-1\right) x}, \\
& f_{2}=\frac{e^{r_{2}} y\left(\beta_{2}+\alpha_{2} x\right)}{\beta_{2}+\alpha_{2} x+\left(e^{r_{2}}-1\right) y},
\end{aligned}
$$

is

$$
V=\left(\begin{array}{cc}
\frac{e^{r_{1}}\left(\beta_{1}+\alpha_{1} y\right)^{2}}{\left(\beta_{1}+\alpha_{1} y+\left(e^{r_{1}}-1\right) x\right)^{2}} \frac{\alpha_{1} e^{r_{1}}\left(e^{r_{1}}-1\right) x^{2}}{\left(\beta_{1}+\alpha_{1} y+\left(e^{r_{1}}-1\right) x\right)^{2}} \\
\frac{\alpha_{2} e^{r_{2}}\left(e^{r_{2}}-1\right) y^{2}}{\left(\beta_{2}+\alpha_{2} x+\left(e^{r_{2}}-1\right) y\right)^{2}} \frac{e^{r_{2}}\left(\beta_{2}+\alpha_{2} x\right)^{2}}{\left(\beta_{2}+\alpha_{2} x+\left(e^{r_{2}}-1\right) y\right)^{2}}
\end{array}\right) .
$$

Now, topological classifications at fixed points $P, Q, R$, and $S$ are explored for the completion of this section.

2.1. Topological Classifications at $P$. The variational matrix at fixed point $P$ is

$$
V_{P}=\left(\begin{array}{cc}
e^{r_{1}} & 0 \\
0 & e^{r_{2}}
\end{array}\right) \text {, }
$$

whose eigenvalues are

$$
\begin{aligned}
& \lambda_{1}=e^{r_{1}}, \\
& \lambda_{2}=e^{r_{2}} .
\end{aligned}
$$

Thus, the topological classifications at $P$ are summarized as Table 1. 
TABle 1: Topological classifications at fixed point $P$.

\begin{tabular}{lc}
\hline Properties & Respective parametric conditions \\
\hline Source & Since $\left|\lambda_{1}\right|=e^{r_{1}}>1$ and $\left|\lambda_{2}\right|=e^{r_{2}}>1$
\end{tabular}

Remark 1. Fixed point $P$ of the discrete Kolmogorov model (3) is never sink, saddle, and nonhyperbolic.

2.2. Topological Classifications at $Q$. The variational matrix at fixed point $Q$ is

$$
V_{Q}=\left(\begin{array}{cc}
e^{r_{1}} & 0 \\
\frac{\alpha_{2}\left(e^{r_{2}}-1\right)}{e^{r_{2}}} & \frac{1}{e^{r_{2}}}
\end{array}\right),
$$

whose eigenvalues are

$$
\begin{aligned}
& \lambda_{1}=e^{r_{1}}, \\
& \lambda_{2}=\frac{1}{e^{r_{2}}} .
\end{aligned}
$$

So, the topological classifications at $Q$ of the discrete Kolmogorov model (3) can be summarized as Table 2.

Remark 2. Fixed point $Q$ of the discrete Kolmogorov model (3) is never sink, source, and nonhyperbolic.

2.3. Topological Classifications at $R$. The variational matrix at fixed point $R$ is

$$
V_{R}=\left(\begin{array}{cc}
\frac{1}{e^{r_{1}}} & \frac{\alpha_{1}\left(e^{r_{1}}-1\right)}{e^{r_{1}}} \\
0 & e^{r_{2}}
\end{array}\right),
$$

whose eigenvalues are

$$
\begin{aligned}
& \lambda_{1}=\frac{1}{e^{r_{1}}}, \\
& \lambda_{2}=e^{r_{2}} .
\end{aligned}
$$

Based on eigenvalues $\lambda_{1,2}$, we will summarize the topological classifications at $Q$ of the discrete-time Kolmogorov model (3) as Table 3.

Remark 3. Fixed point $R$ of the discrete Kolmogorov model (3) is never sink, source, and nonhyperbolic.

2.4. Topological Classifications at S. The variational matrix at fixed point $S$ is

$$
V_{S}=\left(\begin{array}{cc}
\frac{1}{e^{r_{1}}} & \frac{\alpha_{1}\left(e^{r_{1}}-1\right)}{e^{r_{1}}} \\
\frac{\alpha_{2}\left(e^{r_{2}}-1\right)}{e^{r_{2}}} & \frac{1}{e^{r_{2}}}
\end{array}\right),
$$

TABle 2: Topological classifications at fixed point $Q$.

\begin{tabular}{lc}
\hline Properties & Respective parametric conditions \\
\hline Saddle & $\left|\lambda_{1}\right|=e^{r_{1}}>1,\left|\lambda_{2}\right|=\left(1 / e^{r_{2}}\right)<1$
\end{tabular}

TABle 3: Topological classifications at fixed point $R$.

\begin{tabular}{lc}
\hline Properties & Respective parametric conditions \\
\hline Saddle & $\left|\lambda_{1}\right|=\left(1 / e^{r_{1}}\right)<1,\left|\lambda_{2}\right|=e^{r_{2}}>1$ \\
\hline
\end{tabular}

with corresponding characteristic equation of the form,

$$
\lambda^{2}-\tilde{\varrho_{1}} \lambda+\tilde{\varrho_{2}}=0
$$

where

$$
\begin{aligned}
& \tilde{\varrho_{1}}=\frac{e^{r_{1}}+e^{r_{2}}}{e^{r_{1}+r_{2}}}, \\
& \tilde{\varrho_{2}}=\frac{1-\alpha_{1} \alpha_{2}\left(e^{r_{1}}-1\right)\left(e^{r_{2}}-1\right)}{e^{r_{1}+r_{2}}} .
\end{aligned}
$$

Finally, roots of (14) are

$$
\lambda_{1,2}=\frac{-\tilde{\varrho_{1}} \pm \sqrt{\Delta}}{2}
$$

where

$$
\begin{aligned}
\Delta & ={\tilde{\varrho_{1}}}^{2}-4 \tilde{\varrho_{2}}, \\
& =\frac{\left(e^{r_{1}}-e^{r_{2}}\right)^{2}+4 \alpha_{1} \alpha_{2} e^{r_{1}+r_{2}}\left(e^{r_{1}}-1\right)\left(e^{r_{2}}-1\right)}{\left(e^{r_{1}+r_{2}}\right)^{2}}>0 .
\end{aligned}
$$

Since $\Delta>0$, therefore it is important here to note that fixed point $S$ is never stable focus, unstable focus, and nonhyperbolic. So, we will summarize the topological classifications of discrete Kolmogorov model (3) at $S$ as follows.

\section{Exploration of Periodic Points}

In the following proposition, periodic points of prime period-1 of the discrete Kolmogorov model (3) are explored.

Proposition 1. Fixed points $P, Q, R$, and $S$ of the Kolmogorov model (3) are periodic points of prime period-1.

Proof. From (3), defining

$$
F:=\left(f_{1}, f_{2}\right) \text {, }
$$

where $f_{1}$ and $f_{2}$ are defined in (5), after some computations, one gets

$$
\begin{gathered}
\left.F\right|_{P=(0,0)}=P, \\
\left.F\right|_{Q=\left(0, \beta_{2}\right)}=Q, \\
\left.F\right|_{R=\left(\beta_{1}, 0\right)}=R,
\end{gathered}
$$




$$
\left.F\right|_{S=\left(\left(\left(\beta_{1}+\alpha_{1} \beta_{2}\right) /\left(1-\alpha_{1} \alpha_{2}\right)\right),\left(\left(\beta_{2}+\alpha_{2} \beta_{1}\right) /\left(1-\alpha_{1} \alpha_{2}\right)\right)\right)}=S .
$$

From (19)-(22), one can summarize that $P, Q, R$, and $S$ of the Kolmogorov model (3) are periodic points of prime period-1.

In the following proposition, periodic points of period$2,3, \ldots, n$ of the discrete-time Kolmogorov model (3) are explored.
Proposition 2. Fixed points $P, Q, R$, and $S$ of the Kolmogorov model (3) are periodic points of period-2, $3, \ldots, n$.

Proof. After some straightforward computations, from (18), one gets

$$
\begin{aligned}
F^{2} & =\left.\left(\frac{e^{r_{1}} f_{1}\left(\beta_{1}+\alpha_{1} f_{2}\right)}{\beta_{1}+\alpha_{1} f_{2}+\left(e^{r_{1}}-1\right) f_{1}}, \frac{e^{r_{2}} f_{2}\left(\beta_{2}+\alpha_{2} f_{1}\right)}{\beta_{2}+\alpha_{2} f_{1}+\left(e^{r_{2}}-1\right) f_{2}}\right) \Rightarrow F^{2}\right|_{P=(0,0)}=P, \\
F^{3} & =\left.\left(\frac{e^{r_{1}} f_{1}^{2}\left(\beta_{1}+\alpha_{1} f_{2}^{2}\right)}{\beta_{1}+\alpha_{1} f_{2}^{2}+\left(e^{r_{1}}-1\right) f_{1}^{2}}, \frac{e^{r_{2}} f_{2}^{2}\left(\beta_{2}+\alpha_{2} f_{1}^{2}\right)}{\beta_{2}+\alpha_{2} f_{1}^{2}+\left(e^{r_{2}}-1\right) f_{2}^{2}}\right) \Rightarrow F^{3}\right|_{P=(0,0)}=P, \\
& \vdots \\
F^{n} & =\left.\left(\frac{e^{r_{1}} f_{1}^{n-1}\left(\beta_{1}+\alpha_{1} f_{2}^{n-1}\right)}{\beta_{1}+\alpha_{1} f_{2}^{n-1}+\left(e^{r_{1}}-1\right) f_{1}^{n-1}}, \frac{e^{r_{2}} f_{2}^{n-1}\left(\beta_{2}+\alpha_{2} f_{1}^{n-1}\right)}{\beta_{2}+\alpha_{2} f_{1}^{n-1}+\left(e^{r_{2}}-1\right) f_{2}^{n-1}}\right) \Rightarrow F^{n}\right|_{P=(0,0)}=P .
\end{aligned}
$$

From (23), one concludes the required statement. Similarly, one can show that fixed points $Q, R$, and $S$ of the discrete Kolmogorov model (3) are periodic points of period- $2,3, \ldots, n$.

\section{Analysis of Bifurcation}

It is easy to note that there exists no bifurcation at fixed points $P, Q$, and $R$, but in the following subsection, we explore that at $S$ discrete, Kolmogorov model (3) undergoes only flip bifurcation by bifurcation theory [14-20].

4.1. Flip Bifurcation about S. From Table 4, it is noted that equilibrium point $S$ of the discrete Kolmogorov model (3) is nonhyperbolic if $\alpha_{1}=\left(1+e^{r_{1}}+e^{r_{2}}+e^{r_{1}+r_{2}}\right) /\left(\alpha_{2}\left(e^{r_{1}}-\right.\right.$ 1) $\left.\left(e^{r_{2}}-1\right)\right)$. Therefore, eigenvalues of $V_{S}$ at nonhyperbolic condition $\alpha_{1}=\left(1+e^{r_{1}}+e^{r_{2}}+e^{r_{1}+r_{2}}\right) /$ $\left(\alpha_{2}\left(e^{r_{1}}-1\right)\left(e^{r_{2}}-1\right)\right)$ are computed and one gets $\left.\lambda_{1}\right|_{\alpha_{1}=\left(\left(1+e^{r_{1}}+e^{r_{2}}+e^{r_{1}+r_{2}}\right) /\left(\alpha_{2}\left(e^{r_{1}}-1\right)\left(e^{r_{2}}-1\right)\right)\right)}=-1, \quad$ but $\quad \lambda_{2}$ $\left.\right|_{\alpha_{1}=\left(\left(1+e^{r_{1}}+e^{r_{2}}+e^{r_{1}+r_{2}}\right) /\left(\alpha_{2}\left(e^{r_{1}}-1\right)\left(e^{r_{2}}-1\right)\right)\right)}=\quad\left(\left(e^{r_{1}}+e^{r_{2}}+e^{r_{1}+r_{2}}\right) /\right.$ $\left.e^{r_{1}+r_{2}}\right) \neq 1$ or -1 which conclude that at fixed point $S$, Kolmogorov model (3) undergoes flip bifurcation if $\left(r_{1}, r_{2}, \alpha_{1}, \alpha_{2}, \beta_{1}, \beta_{2}\right)$ are located in the following set:

$$
\left.\mathscr{F}\right|_{S}=\left\{\left(r_{1}, r_{2}, \alpha_{1}, \alpha_{2}, \beta_{1}, \beta_{2}\right), \quad \alpha_{1}=\frac{1+e^{r_{1}}+e^{r_{2}}+e^{r_{1}+r_{2}}}{\alpha_{2}\left(e^{r_{1}}-1\right)\left(e^{r_{2}}-1\right)}\right\} .
$$

Hereafter, in the following, we will present comprehensive flip bifurcation analysis at $S$ of the Kolmogorov model (3).
Theorem 1. If $\left.\left(r_{1}, r_{2}, \alpha_{1}, \alpha_{2}, \beta_{1}, \beta_{2}\right) \in \mathscr{F}\right|_{S}$, then the discrete Kolmogorov model (3) undergoes the flip bifurcation.

Proof. It is recalled from Table 4 that if $\alpha_{1}=\left(1+e^{r_{1}}+e^{r_{2}}+e^{r_{1}+r_{2}}\right) /\left(\alpha_{2}\left(e^{r_{1}}-1\right)\left(e^{r_{2}}-1\right)\right)$, then $S$ is nonhyperbolic where $\Delta=\left(\left(\left(e^{r_{1}}-e^{r_{2}}\right)^{2}+4 \alpha_{1} \alpha_{2} e^{r_{1}+r_{2}}\left(e^{r_{1}}-\right.\right.\right.$ 1) $\left.\left.\left(e^{r_{2}}-1\right)\right) /\left(e^{r_{1}+r_{2}}\right)^{2}\right)>0$. Moreover, at $\alpha_{1}=((1+$ $\left.\left.e^{r_{1}}+e^{r_{2}}+e^{r_{1}+r_{2}}\right) /\left(\alpha_{2}\left(e^{r_{1}}-1\right)\left(e^{r_{2}}-1\right)\right)\right)$, one gets $\left.\lambda_{1}\right|_{\alpha_{1}}$ $=\left(\left(1+e^{r_{1}}+e^{r_{2}}+e^{r_{1}+r_{2}}\right) /\left(\alpha_{2}\left(e^{r_{1}}-1\right)\left(e^{r_{2}}-1\right)\right)\right)=-1$, where as $\left.\lambda_{2}\right|_{\alpha_{1}}=\left(\left(1+e^{r_{1}}+e^{r_{2}}+e^{r_{1}+r_{2}}\right) /\left(\alpha_{2}\left(e^{r_{1}}-1\right)\left(e^{r_{2}}-\right.\right.\right.$ $1)))=\left(\left(e^{r_{1}}+e^{r_{2}}+e^{r_{1}+r_{2}}\right) / e^{r_{1}+r_{2}}\right) \neq 1$ or -1 , which gives the existence of flip bifurcation at $S$ by choosing $\alpha_{1}$ as a bifurcation parameter. So, if $\alpha_{1}$ varies in a neighborhood of $\alpha_{1}^{*}$, then model (3) takes the form,

$$
\begin{aligned}
& x_{n+1}=\frac{e^{r_{1}} x_{n}\left(\beta_{1}+\left(\alpha_{1}^{*}+\epsilon\right) y_{n}\right)}{\beta_{1}+\left(\alpha_{1}^{*}+\epsilon\right) y_{n}+\left(e^{r_{1}}-1\right) x_{n}}, \\
& y_{n+1}=\frac{e^{r_{2}} y_{n}\left(\beta_{2}+\alpha_{2} x_{n}\right)}{\beta_{2}+\alpha_{2} x_{n}+\left(e^{r_{2}}-1\right) y_{n}} .
\end{aligned}
$$

Now, it is noted that by using transformation,

$$
\begin{aligned}
& u_{n}=x_{n}-x^{*}, \\
& v_{n}=y_{n}-y^{*},
\end{aligned}
$$

one can transform $S$ to $P$ where (25) takes the following form:

$$
\begin{aligned}
u_{n+1}= & \widehat{D_{11}} u_{n}+\widehat{D_{12}} v_{n}+\widehat{D_{13}} u_{n}^{2}+\widehat{D_{14}} u_{n} v_{n}+\widehat{D_{15}} v_{n}^{2} \\
& +\gamma_{01} u_{n} \epsilon+\gamma_{02} v_{n} \epsilon+\gamma_{03} u_{n}^{2} \epsilon+\gamma_{04} u_{n} v_{n} \epsilon+\gamma_{05} v_{n}^{2} \epsilon, \\
v_{n+1}= & \widehat{D_{21}} u_{n}+\widehat{D_{22}} v_{n}+\widehat{D_{23}} u_{n}^{2}+\widehat{D_{24}} u_{n} v_{n}+\widehat{D_{25}} v_{n}^{2},
\end{aligned}
$$


TABle 4: Topological classifications at fixed point $S$.

\begin{tabular}{lc}
\hline Properties & Respective parametric conditions \\
\hline Stable node & $\alpha_{1}>\left(\left(1+e^{r_{1}}+e^{r_{2}}+e^{r_{1}+r_{2}}\right) /\left(\alpha_{2}\left(e^{r_{1}}-1\right)\left(e^{r_{2}}-1\right)\right)\right)$ \\
Unstable node & $0<\alpha_{1}<\left(\left(1+e^{r_{1}}+e^{r_{2}}+e^{r_{1}+r_{2}}\right) /\left(\alpha_{2}\left(e^{r_{1}}-1\right)\left(e^{r_{2}}-1\right)\right)\right)$ \\
Nonhyperbolic & $\alpha_{1}=\left(\left(1+e^{r_{1}}+e^{r_{2}}+e^{r_{1}+r_{2}}\right) /\left(\alpha_{2}\left(e^{r_{1}}-1\right)\left(e^{r_{2}}-1\right)\right)\right)$ \\
\hline
\end{tabular}

where

$$
\begin{aligned}
& \widehat{D_{11}}=\frac{e^{r_{1}}\left(\beta_{1}+\alpha_{1}^{*} y^{*}\right)^{2}}{\left(\beta_{1}+\alpha_{1}^{*} y^{*}+\left(e^{r_{1}}-1\right) x^{*}\right)^{2}}, \\
& \widehat{D_{12}}=\frac{e^{r_{1}} \alpha_{1}^{*}\left(e^{r_{1}}-1\right) x^{* 2}}{\left(\beta_{1}+\alpha_{1}^{*} y^{*}+\left(e^{r_{1}}-1\right) x^{*}\right)^{2}}, \\
& \widehat{D_{13}}=-\frac{e^{r_{1}}\left(e^{r_{1}}-1\right)\left(\beta_{1}+\alpha_{1}^{*} y^{*}\right)^{2}}{\left(\beta_{1}+\alpha_{1}^{*} y^{*}+\left(e^{r_{1}}-1\right) x^{*}\right)^{3}} \\
& \widehat{D_{14}}=\frac{e^{r_{1}} \alpha_{1}^{*}\left(e^{r_{1}}-1\right)\left(\beta_{1}+\alpha_{1}^{*} y^{*}\right) x^{*}}{\left(\beta_{1}+\alpha_{1}^{*} y^{*}+\left(e^{r_{1}}-1\right) x^{*}\right)^{3}}, \\
& \widehat{D_{15}}=-\frac{\alpha_{1}^{* 2} e^{r_{1}}\left(e^{r_{1}}-1\right) x^{* 2}}{\left(\beta_{1}+\alpha_{1}^{*} y^{*}+\left(e^{r_{1}}-1\right) x^{*}\right)^{3}}, \\
& \gamma_{01}=\frac{2 e^{r_{1}}\left(\beta_{1}+\alpha_{1}^{*} y^{*}\right) y^{*}}{\left(\beta_{1}+\alpha_{1}^{*} y^{*}+\left(e^{r_{1}}-1\right) x^{*}\right)^{2}}, \\
& \gamma_{02}=\frac{e^{r_{1}}\left(e^{r_{1}}-1\right) x^{* 2}}{\left(\beta_{1}+\alpha_{1}^{*} y^{*}+\left(e^{r_{1}}-1\right) x^{*}\right)^{2}}, \\
& \gamma_{03}=-\frac{2 e^{r_{1}}\left(e^{r_{1}}-1\right)\left(\beta_{1}+\alpha_{1}^{*} y^{*}\right) y^{*}}{\left(\beta_{1}+\alpha_{1}^{*} y^{*}+\left(e^{r_{1}}-1\right) x^{*}\right)^{3}}, \\
& \gamma_{04}=\frac{e^{r_{1}}\left(e^{r_{1}}-1\right)\left(\beta_{1}+2 \alpha_{1}^{*} y^{*}\right) x^{*}}{\left(\beta_{1}+\alpha_{1}^{*} y^{*}+\left(e^{r_{1}}-1\right) x^{*}\right)^{3}}, \\
& \gamma_{05}=-\frac{2 e^{r_{1}}\left(e^{r_{1}}-1\right) x^{* 2} \alpha_{1}^{*}}{\left(\beta_{1}+\alpha_{1}^{*} y^{*}+\left(e^{r_{1}}-1\right) x^{*}\right)^{3}}, \\
& \widehat{D_{21}}=\frac{\alpha_{2} e^{r_{2}}\left(e^{r_{2}}-1\right) y^{* 2}}{\left(\beta_{2}+\alpha_{2} x^{*}+\left(e^{r_{2}}-1\right) y^{*}\right)^{2}}, \\
& \widehat{D_{22}}=\frac{e^{r_{2}}\left(\beta_{2}+\alpha_{2} x^{*}\right)^{2}}{\left(\beta_{2}+\alpha_{2} x^{*}+\left(e^{r_{2}}-1\right) y^{*}\right)^{2}}, \\
& \widehat{D_{23}}=-\frac{\alpha_{2}^{2} e^{r_{2}}\left(e^{r_{2}}-1\right) y^{* 2}}{\left(\beta_{2}+\alpha_{2} x^{*}+\left(e^{r_{2}}-1\right) y^{*}\right)^{3}}, \\
& \widehat{D_{24}}=\frac{\alpha_{2} e^{r_{2}}\left(e^{r_{2}}-1\right)\left(\beta_{2}+\alpha_{2} x^{*}\right) y^{*}}{\left(\beta_{2}+\alpha_{2} x^{*}+\left(e^{r_{2}}-1\right) y^{*}\right)^{3}}, \\
& \widehat{D_{25}}=-\frac{e^{r_{2}}\left(e^{r_{2}}-1\right)\left(\beta_{2}+\alpha_{2} x^{*}\right)^{2}}{\left(\beta_{2}+\alpha_{2} x^{*}+\left(e^{r_{2}}-1\right) y^{*}\right)^{3}} .
\end{aligned}
$$


Now, (27) takes the following form:

where

$$
\left(\begin{array}{c}
X_{n+1} \\
Y_{n+1}
\end{array}\right)=\left(\begin{array}{cc}
-1 & 0 \\
0 & \lambda_{2}
\end{array}\right)\left(\begin{array}{l}
X_{n} \\
Y_{n}
\end{array}\right)+\left(\begin{array}{c}
\widehat{P}(\epsilon) \\
\widehat{Q}(\epsilon)
\end{array}\right),
$$

$$
\begin{aligned}
& \widehat{P}(\epsilon)=\frac{\widehat{D_{13}}\left(\lambda_{2}-\widehat{D_{11}}\right)-\widehat{D_{12} D_{23}}}{\widehat{D_{12}}\left(1+\lambda_{2}\right)} u_{n}^{2}+\frac{\widehat{D_{14}}\left(\lambda_{2}-\widehat{D_{11}}\right)-\widehat{D_{12}} \widehat{D_{24}}}{\widehat{D_{12}}\left(1+\lambda_{2}\right)} u_{n} v_{n} \\
& +\frac{\widehat{D_{15}}\left(\lambda_{2}-\widehat{D_{11}}\right)-\widehat{D_{12} D_{25}}}{\widehat{D_{12}}\left(1+\lambda_{2}\right)} v_{n}^{2}+\frac{\gamma_{01}\left(\lambda_{2}-\widehat{D_{11}}\right)}{\widehat{D_{12}}\left(1+\lambda_{2}\right)} u_{n} \epsilon+\frac{\gamma_{02}\left(\lambda_{2}-\widehat{D_{11}}\right)}{\widehat{D_{12}}\left(1+\lambda_{2}\right)} v_{n} \epsilon \\
& +\frac{\gamma_{03}\left(\lambda_{2}-\widehat{D_{11}}\right)}{\widehat{D_{12}}\left(1+\lambda_{2}\right)} u_{n}^{2} \epsilon+\frac{\gamma_{04}\left(\lambda_{2}-\widehat{D_{11}}\right)}{\widehat{D_{12}}\left(1+\lambda_{2}\right)} u_{n} v_{n} \epsilon+\frac{\gamma_{05}\left(\lambda_{2}-\widehat{D_{11}}\right)}{\widehat{D_{12}}\left(1+\lambda_{2}\right)} v_{n}^{2} \epsilon, \\
& \widehat{Q}(\epsilon)=\frac{\widehat{D_{13}}\left(1+\widehat{D_{11}}\right)+\widehat{D_{12} D_{23}}}{\widehat{D_{12}}\left(1+\lambda_{2}\right)} u_{n}^{2}+\frac{\widehat{D_{14}}\left(1+\widehat{D_{11}}\right)+\widehat{D_{12}} \widehat{D_{24}}}{\widehat{D_{12}}\left(1+\lambda_{2}\right)} u_{n} v_{n} \\
& +\frac{\widehat{D_{15}}\left(1+\widehat{D_{11}}\right)+\widehat{D_{12} D_{25}}}{\widehat{D_{12}}\left(1+\lambda_{2}\right)} v_{n}^{2}+\frac{\gamma_{01}\left(1+\widehat{D_{11}}\right)}{\widehat{D_{12}}\left(1+\lambda_{2}\right)} u_{n} \epsilon+\frac{\gamma_{02}\left(1+\widehat{D_{11}}\right)}{\widehat{D_{12}}\left(1+\lambda_{2}\right)} v_{n} \epsilon \\
& +\frac{\gamma_{03}\left(1+\widehat{D_{11}}\right)}{\widehat{D_{12}}\left(1+\lambda_{2}\right)} u_{n}^{2} \epsilon+\frac{\gamma_{04}\left(1+\widehat{D_{11}}\right)}{\widehat{D_{12}}\left(1+\lambda_{2}\right)} u_{n} v_{n} \epsilon+\frac{\gamma_{05}\left(1+\widehat{D_{11}}\right)}{\widehat{D_{12}}\left(1+\lambda_{2}\right)} v_{n}^{2} \epsilon \\
& u_{n}^{2}={\widehat{D_{12}}}^{2}\left(X_{n}^{2}+2 X_{n} Y_{n}+Y_{n}^{2}\right) \\
& \left.u_{n} v_{n}=-\widehat{D_{12}}\left(1+\widehat{D_{11}}\right) X_{n}^{2}+\widehat{\widehat{D_{12}}}\left(\lambda_{2}-\widehat{D_{11}}\right)-\widehat{D_{12}}\left(1+\widehat{D_{11}}\right)\right) X_{n} Y_{n} \\
& +\widehat{D_{12}}\left(\lambda_{2}-\widehat{D_{11}}\right) Y_{n}^{2} \\
& v_{n}^{2}=\left(1+\widehat{D_{11}}\right)^{2} X_{n}^{2}-2\left(1+\widehat{D_{11}}\right)\left(\lambda_{2}-\widehat{D_{11}}\right) X_{n} Y_{n}+\left(\lambda_{2}-\widehat{D_{11}}\right)^{2} Y_{n}^{2}, \\
& u_{n} \epsilon=\widehat{D_{12}} X_{n} \epsilon+\widehat{D_{12}} Y_{n} \epsilon, \\
& v_{n} \epsilon=\left(-1-\widehat{D_{11}}\right) X_{n} \epsilon+\left(\lambda_{2}-\widehat{D_{11}}\right) Y_{n} \epsilon \\
& u_{n}^{2} \epsilon={\widehat{D_{12}}}^{2}\left(X_{n}^{2} \epsilon+2 X_{n} Y_{n} \epsilon+Y_{n}^{2} \epsilon\right) \\
& u_{n} v_{n} \epsilon=-\widehat{D_{12}}\left(1+\widehat{D_{11}}\right) X_{n}^{2} \epsilon+\left(\widehat{D_{12}}\left(\lambda_{2}-\widehat{D_{11}}\right)-\widehat{D_{12}}\left(1+\widehat{D_{11}}\right)\right) X_{n} Y_{n} \epsilon \\
& +\widehat{D_{12}}\left(\lambda_{2}-\widehat{D_{11}}\right) Y_{n}^{2} \epsilon \\
& v_{n}^{2} \epsilon=\left(1+\widehat{D_{11}}\right)^{2} X_{n}^{2} \epsilon-2\left(1+\widehat{D_{11}}\right)\left(\lambda_{2}-\widehat{D_{11}}\right) X_{n} Y_{n} \epsilon+\left(\lambda_{2}-\widehat{D_{11}}\right)^{2} Y_{n}^{2} \epsilon
\end{aligned}
$$

by

$$
\left(\begin{array}{c}
u_{n} \\
v_{n}
\end{array}\right)=\left(\begin{array}{cc}
\widehat{D_{12}} & \widehat{D_{12}} \\
-1-\widehat{D_{11}} & \lambda_{2}-\widehat{D_{11}}
\end{array}\right)\left(\begin{array}{l}
X_{n} \\
Y_{n}
\end{array}\right) .
$$

Now, for (29), center manifold $M^{c} P$ about $P$ is examined in a small neighborhood of $\epsilon$, and therefore $M^{c} P$ can be expressed as the following expression: 


$$
M^{c} P=\left\{\left(X_{n}, Y_{n}\right): \quad Y_{n}=\widehat{h_{0}} \epsilon+\widehat{h_{1}} X_{n}^{2}+\widehat{h_{2}} X_{n} \epsilon+\widehat{h_{3}} \epsilon^{3}+O\left(\left(\left|X_{n}\right|+|\epsilon|\right)^{3}\right)\right\} .
$$

Therefore, the computation yields

$\widehat{h_{0}}=0$,

$$
\begin{aligned}
\widehat{h_{1}}= & \frac{\left(1+\widehat{D_{11}}\right)\left[\widehat{D_{12}} \widehat{D_{13}}-\widehat{D_{14}}\left(1+\widehat{D_{11}}\right)-\widehat{D_{12}} \widehat{D_{24}}\right]}{1-\lambda_{2}^{2}}+\frac{{\widehat{D_{12}}}^{2} \widehat{D_{23}}}{1-\lambda_{2}^{2}} \\
& +\frac{\left(1+\widehat{D_{11}}\right)^{2}\left[\widehat{D_{15}}\left(1+\widehat{D_{11}}\right)+\widehat{D_{12}} \widehat{D_{25}}\right]}{\widehat{D_{12}}\left(1-\lambda_{2}^{2}\right)}
\end{aligned}
$$$$
\widehat{h_{2}}=\frac{\widehat{D_{12}} \gamma_{01}\left(1+\widehat{D_{11}}\right)-\gamma_{02}\left(1+\widehat{D_{11}}\right)^{2}}{\widehat{D_{12}}\left(1-\lambda_{2}^{2}\right)}
$$

$\widehat{h_{3}}=0$.
Thus, the map (29) restricting to $M^{c} P$ is

$$
\begin{aligned}
f\left(x_{n}\right)= & -x_{n}+\widehat{v_{1}} x_{n}^{2}+\widehat{v_{2}} x_{n} \epsilon+\widehat{v_{3}} x_{n}^{2} \epsilon+\widehat{v_{4}} x_{n} \epsilon^{2} \\
& +\widehat{v_{5}} x_{n}^{3}+O\left(\left(\left|X_{n}\right|+|\epsilon|\right)^{4}\right),
\end{aligned}
$$

$$
\begin{aligned}
& \widehat{v_{1}}=\frac{1}{1+\lambda_{2}}\left[\widehat{D_{12}} \widehat{D_{13}}\left(\lambda_{2}-\widehat{D_{11}}\right)-\left(1+\widehat{D_{11}}\right)\left(\widehat{D_{14}}\left(\lambda_{2}-\widehat{D_{11}}\right)-\widehat{D_{12}} \widehat{D_{24}}\right)-{\widehat{D_{12}}}^{2} \widehat{D_{23}}\right. \\
& \left.-\widehat{D_{25}}\left(1+\widehat{D_{11}}\right)^{2}+\frac{\widehat{D_{15}}\left(\lambda_{2}-\widehat{D_{11}}\right)\left(1+\widehat{D_{11}}\right)^{2}}{\widehat{D_{12}}}\right] \\
& \widehat{v_{2}}=\frac{1}{1+\lambda_{2}}\left[\gamma_{01}\left(\lambda_{2}-\widehat{D_{11}}\right)-\frac{\gamma_{02}\left(\lambda_{2}-\widehat{D_{11}}\right)\left(1+\widehat{D_{11}}\right)}{\widehat{D_{12}}}\right] \\
& \widehat{v_{3}}=\frac{1}{1+\lambda_{2}}\left[2 \widehat{D_{12}} \widehat{h_{2}} \widehat{D_{13}}\left(\lambda_{2}-\widehat{D_{11}}\right)-2 \widehat{h_{2}}{\widehat{D_{12}}}^{2} \widehat{D_{23}}\right. \\
& +\left(\widehat{h_{2}}\left(\lambda_{2}-\widehat{D_{11}}\right)-\widehat{h_{2}}\left(1+\widehat{D_{11}}\right)\right)\left(\widehat{D_{14}}\left(\lambda_{2}-\widehat{D_{11}}\right)-\widehat{D_{12}} \widehat{D_{24}}\right)+\widehat{h_{1}} \gamma_{01}\left(\lambda_{2}-\widehat{D_{11}}\right) \\
& -\frac{2 \widehat{h_{2}}\left(1+\widehat{D_{11}}\right)\left(\lambda_{2}-\widehat{D_{11}}\right)\left(\widehat{D_{15}}\left(\lambda_{2}-\widehat{D_{11}}\right)-\widehat{D_{12}} \widehat{D_{25}}\right)}{\widehat{D_{12}}}+\frac{\widehat{h_{1}} \gamma_{02}\left(\lambda_{2}-\widehat{D_{11}}\right)^{2}}{\widehat{D_{12}}}+\widehat{D_{12}} \gamma_{03}\left(\lambda_{2}-\widehat{D_{11}}\right) \\
& \left.-\gamma_{04}\left(1+\widehat{D_{11}}\right)\left(\lambda_{2}-\widehat{D_{11}}\right)+\gamma_{05} \frac{\left(1+\widehat{D_{11}}\right)^{2}}{\widehat{D_{12}}}\left(\lambda_{2}-\widehat{D_{11}}\right)\right] \text {, } \\
& \widehat{v_{4}}=\frac{\widehat{h_{2}}}{1+\lambda_{2}}\left[\gamma_{01}\left(\lambda_{2}-\widehat{D_{11}}\right)+\gamma_{02} \frac{\left(\lambda_{2}-\widehat{D_{11}}\right)^{2}}{\widehat{D_{12}}}\right] \text {, } \\
& \widehat{v_{5}}=\frac{1}{1+\lambda_{2}}\left[2 \widehat{h_{1}} \widehat{D_{12}}\left(\widehat{D_{13}}\left(\lambda_{2}-\widehat{D_{11}}\right)-\widehat{D_{12}} \widehat{D_{23}}\right)\right. \\
& +\left(\widehat{h_{1}}\left(\lambda_{2}-\widehat{D_{11}}\right)-\widehat{h_{1}}\left(1+\widehat{D_{11}}\right)\right)\left(\widehat{D_{14}}\left(\lambda_{2}-\widehat{D_{11}}\right)-\widehat{D_{12}} \widehat{D_{24}}\right) \\
& \left.-\frac{2 \widehat{h_{1}}\left(\lambda_{2}-\widehat{D_{11}}\right)\left(1+\widehat{D_{11}}\right)}{\widehat{D_{12}}}\left(\widehat{D_{15}}\left(\lambda_{2}-\widehat{D_{11}}\right)-\widehat{D_{12}} \widehat{D_{25}}\right)\right] .
\end{aligned}
$$


In order to show (34) undergoes flip bifurcation, it is required that $Q_{1}, Q_{2}$ must be nonzero, i.e.,

$$
\begin{aligned}
& Q_{1}=\left.\left(\frac{\partial^{2} f}{\partial x_{n} \partial \epsilon}+\frac{1}{2} \frac{\partial f}{\partial \epsilon} \frac{\partial^{2} f}{\partial x_{n}^{2}}\right)\right|_{P}, \\
& Q_{2}=\left(\frac{1}{6} \frac{\partial^{3} f}{\partial x_{n}^{3}}+\left(\frac{1}{2} \frac{\partial^{2} f}{\partial x_{n}^{2}}\right)^{2}\right) \mid P .
\end{aligned}
$$

After calculating, one gets

$$
\begin{aligned}
Q_{1}= & \frac{\alpha_{2}\left(e^{r_{1}}-1\right)\left(e^{2 r_{2}}-1\right)}{e^{r_{1}}+e^{r_{2}}+2 e^{r_{1}+r_{2}}}\left[\frac{2\left(\beta_{2}+\alpha_{2} \beta_{1}\right)}{\beta_{1} \alpha_{2}\left(e^{r_{1}}-1\right)\left(e^{r_{2}}-1\right)+\beta_{2}\left(1+e^{r_{1}}+e^{r_{2}}+e^{r_{1}+r_{2}}\right)}-\frac{1+e^{r_{2}}}{1+e^{r_{1}}+e^{r_{2}}+e^{r_{1}+r_{2}}}\right] \neq 0, \\
Q_{2}= & \frac{2 \widehat{h_{1}}\left(1+e^{r_{1}}+e^{r_{2}}+e^{r_{1}+r_{2}}\right)}{e^{r_{1}}+e^{r_{2}}+e^{r_{1}+r_{2}}}\left[\frac{e^{r_{2}}\left(e^{r_{1}}-1\right)\left(e^{r_{2}}-1\right)}{\beta_{1} \alpha_{2}\left(e^{r_{1}}-1\right)\left(e^{r_{2}}-1\right)+\beta_{2}\left(1+e^{r_{1}}+e^{r_{2}}+e^{r_{1}+r_{2}}\right)}\right. \\
& \times\left(\frac{2\left(1-e^{r_{1}}\right)}{e^{r_{1}}}+\frac{1+e^{r_{2}}}{e^{r_{2}}}-\frac{1+e^{r_{1}}}{e^{r_{1}}}+\frac{2\left(1+e^{r_{1}}\right)\left(1+e^{r_{2}}\right)}{e^{r_{2}}\left(e^{r_{1}}-1\right)}\right) \\
& \left.+\frac{e^{r_{1}}}{\beta_{2}+\alpha_{2} \beta_{1}}\left(\frac{2\left(1+e^{r_{1}}+e^{r_{2}}+e^{r_{1}+r_{2}}\right)}{e^{r_{1}}\left(e^{r_{2}}-1\right)}-\frac{1+e^{r_{2}}}{e^{r_{2}}}-\frac{1+e^{r_{1}}}{e^{r_{1}}}-\frac{2\left(1+e^{r_{1}}\right)\left(e^{2 r_{2}}-1\right)}{e^{r_{2}}\left(1+e^{r_{1}}+e^{r_{2}}+e^{r_{1}+r_{2}}\right)}\right)\right] \\
& +\frac{4}{\left(e^{r_{1}}+e^{r_{2}}+e^{r_{1}+r_{2}}\right)^{2}}\left[\frac{e^{r_{2}}\left(1+e^{r_{2}}\right)\left(e^{r_{1}}-1\right)\left(1+e^{r_{1}}+e^{r_{2}}+e^{r_{1}+r_{2}}\right)}{\left.e^{r_{1}} \alpha_{2}\left(e^{r_{1}}-1\right)\left(e^{r_{2}}-1\right)+\beta_{2}\left(1+e^{r_{1}}+e^{r_{2}}+e^{r_{1}+r_{2}}\right)\right)}\right. \\
& \left.+\frac{1}{\beta_{2}+\alpha_{2} \beta_{1}}\left(\left(1+e^{r_{1}}\right)\left(1+e^{r_{1}}+e^{r_{2}}+e^{r_{1}+r_{2}}\right)+\left(1+e^{r_{2}}\right)^{2}-\frac{\left(1+e^{r_{1}}+e^{r_{2}}+e^{r_{1}+r_{2}}\right)^{2}}{e^{r_{2}}-1}\right)\right]^{2},
\end{aligned}
$$

where

$$
\begin{aligned}
\widehat{h_{1}}= & \frac{2 e^{r_{1}+r_{2}}\left(1+e^{r_{1}}\right)\left(1+e^{r_{1}}+e^{r_{2}}+e^{r_{1}+r_{2}}\right)}{\left(e^{r_{1}+r_{2}}\right)^{2}-\left(e^{r_{1}}+e^{r_{2}}+e^{r_{1}+r_{2}}\right)^{2}}\left[\frac{-1}{\beta_{2}+\alpha_{2} \beta_{1}}\left(\alpha_{2}+1\right)\right. \\
& \left.+\frac{e^{2 r_{2}}-e^{2\left(r_{1}+r_{2}\right)}}{e^{2 r_{1}}\left(\beta_{1} \alpha_{2}\left(e^{r_{1}}-1\right)\left(e^{r_{2}}-1\right)+\left(1+e^{r_{1}}+e^{r_{2}}+e^{r_{1}+r_{2}}\right) \beta_{2}\right)}\right] \\
& -\frac{2 e^{r_{1}+r_{2}}\left(1+e^{r_{1}}+e^{r_{2}}+e^{r_{1}+r_{2}}\right)}{\alpha_{2}\left(e^{r_{1}}-1\right)\left(e^{r_{2}}-1\right)^{2}\left(\beta_{2}+\alpha_{2} \beta_{1}\right)\left(e^{2\left(r_{1}+r_{2}\right)}-\left(e^{r_{1}}+e^{r_{2}}+e^{r_{1}+r_{2}}\right)^{2}\right)} \\
& +\frac{2\left(1+e^{r_{1}}\right)^{2} e^{r_{1}+r_{2}}}{\left(e^{2\left(r_{1}+r_{2}\right)}-\left(e^{r_{1}}+e^{r_{2}}+e^{r_{1}+r_{2}}\right)^{2}\right) e^{2 r_{2}}}\left[\frac{\left(e^{2 r_{1}}\left(\beta_{1} \alpha_{2}\left(e^{r_{1}}-1\right)\left(e^{r_{2}}-1\right)+\left(1+e^{r_{1}}+e^{r_{2}}+e^{r_{1}+r_{2}}\right) \beta_{2}\right)\right.}{1+e^{r_{1}}}\right. \\
& \left.-\frac{\alpha_{2}\left(e^{r_{1}}-1\right)\left(e^{r_{2}}-1\right)\left(\beta_{2}+\alpha_{2} \beta_{1}\right)}{e^{2}}\right] .
\end{aligned}
$$


In view of (38) and (39), if $Q_{2} \neq 0$, then as $\left.\left(r_{1}, r_{2}, \alpha_{1}, \alpha_{2}, \beta_{1}, \beta_{2}\right) \in \mathscr{F}\right|_{S}$, discrete Kolmogorov model (3) undergoes a flip bifurcation. Additionally, period-2 points from $S$ are stable (respectively unstable), if $Q_{2}>0$ (respectively $Q_{2}<0$ ).

\section{Chaos Control}

This section is purely dedicated for the exploration of chaos control in the sense of state feedback control method [21, 22]. After adding control force $u_{t}$, discrete Kolmogorov model (3) takes the form,

$$
\begin{aligned}
& x_{n+1}=\frac{e^{r_{1}} x_{n}\left(\beta_{1}+\alpha_{1} y_{n}\right)}{\left(\beta_{1}+\alpha_{1} y_{n}\right)+\left(e^{r_{1}}-1\right) x_{n}}+u_{n}, \\
& y_{n+1}=\frac{e^{r_{2}} y_{n}\left(\beta_{2}+\alpha_{2} x_{n}\right)}{\left(\beta_{2}+\alpha_{2} x_{n}\right)+\left(e^{r_{2}}-1\right) y_{n}},
\end{aligned}
$$

where $\quad u_{n}=-l_{1}\left(x_{n}-\left(\left(\beta_{1}+\alpha_{1} \beta_{2}\right) /\left(1-\alpha_{1} \alpha_{2}\right)\right)\right)-l_{2}\left(y_{n}-\right.$ $\left.\left(\left(\beta_{2}+\alpha_{2} \beta_{1}\right) /\left(1-\alpha_{1} \alpha_{2}\right)\right)\right)$. It is noted here that $l_{1}, l_{2}$ denotes feedback gains. Moreover, $V_{S}$ for the controlled system (40) is

$$
V_{S}^{C}=\left(\begin{array}{cc}
\frac{1}{e^{r_{1}}}-l_{1} & \frac{\alpha_{1}\left(e^{r_{1}}-1\right)}{e^{r_{1}}}-l_{2} \\
\frac{\alpha_{2}\left(e^{r_{2}}-1\right)}{e^{r_{2}}} & \frac{1}{e^{r_{2}}}
\end{array}\right) .
$$

If $\lambda_{1,2}$ denotes the characteristic roots of $V_{S}^{C}$ at $S$, then

$$
\begin{aligned}
\lambda_{1}+\lambda_{2}= & -l_{1}+\frac{1}{e^{r_{1}}}+\frac{1}{e^{r_{2}}}, \\
\lambda_{1} \lambda_{2}= & -\frac{1}{e^{r_{1}}} l_{1}+\frac{\alpha_{2}\left(e^{r_{2}}-1\right)}{e^{r_{2}}} l_{2} \\
& +\frac{1}{e^{r_{1}+r_{2}}}-\frac{\alpha_{1} \alpha_{2}\left(e^{r_{1}}-1\right)\left(e^{r_{2}}-1\right)}{e^{r_{1}+r_{2}}} .
\end{aligned}
$$

Now, the answer of $\lambda_{1}= \pm 1$ and $\lambda_{1} \lambda_{2}=1$ determines the strains of marginal stability, and these prerequisites give that $\left|\lambda_{1,2}\right|<1$. If $\lambda_{1} \lambda_{2}=1$, then from (43), one gets

$$
L_{1}:-\frac{1}{e^{r_{1}}} l_{1}+\frac{\alpha_{2}\left(e^{r_{2}}-1\right)}{e^{r_{2}}} l_{2}+\frac{1}{e^{r_{1}+r_{2}}}-\frac{\alpha_{1} \alpha_{2}\left(e^{r_{1}}-1\right)\left(e^{r_{2}}-1\right)}{e^{r_{1}+r_{2}}}-1=0 .
$$

If $\lambda_{1}=1$, then from (42) and (43), one gets

$$
\begin{gathered}
L_{2}:\left(1-\frac{1}{e^{r_{1}}}\right) l_{1}+\frac{\alpha_{2}\left(e^{r_{2}}-1\right)}{e^{r_{2}}} l_{2}+\frac{1}{e^{r_{1}+r_{2}}}-\frac{1}{e^{r_{1}}} \\
-\frac{1}{e^{r_{2}}}-\frac{\alpha_{1} \alpha_{2}\left(e^{r_{1}}-1\right)\left(e^{r_{2}}-1\right)}{e^{r_{1}+r_{2}}}+1=0 .
\end{gathered}
$$

Finally, if $\lambda_{1}=-1$, then from (42) and (43), one gets

$$
\begin{aligned}
L_{3}: & -\left(1+\frac{1}{e^{r_{1}}}\right) l_{1}+\frac{\alpha_{2}\left(e^{r_{2}}-1\right)}{e^{r_{2}}} l_{2}+\frac{1}{e^{r_{1}+r_{2}}}+\frac{1}{e^{r_{1}}}+\frac{1}{e^{r_{2}}} \\
& -\frac{\alpha_{1} \alpha_{2}\left(e^{r_{1}}-1\right)\left(e^{r_{2}}-1\right)}{e^{r_{1}+r_{2}}}+1=0 .
\end{aligned}
$$

Therefore, from (44)-(46), lines $L_{1}, L_{2}$, and $L_{3}$ in $\left(l_{1}, l_{2}\right)$-plane give the triangular region, which further gives the fact that $\left|\lambda_{1,2}\right|<1$.

\section{Numerical Simulations}

We present numerical simulations to validate the corresponding obtained results. For instance, if $r_{1}=5.25$, $r_{2}=5.3, \alpha_{2}=0.4, \beta_{1}=2.9, \beta_{2}=2.9$, then from nonhyperbolic condition, one gets $\alpha_{1}=\left(\left(1+e^{r_{1}}+e^{r_{2}}+e^{r_{1}+r_{2}}\right) /\right.$ $\left.\left(\alpha_{2}\left(e^{r_{1}}-1\right)\left(e^{r_{2}}-1\right)\right)\right)=2.5517238126301827$. From theoretical discussion, $S=(4.284090909090909$, 4.613636363636363) of the discrete Kolmogorov model (3) is a stable node if $\alpha_{1}>2.5517238126301827$. So, if one chose the bifurcation value $\alpha_{1}=2.652>2.5517238126301827$, then it is clear from Figure 1(a) that fixed point $S=(4.284090909090909,4.613636363636363)$ of Kolmogorov model (3) is a stable node. Moreover, for some more parametric values, if $\alpha_{1}=2.6587,2.7,2.765>$ 2.5517238126301827 , then Figures 1(b)-1(d) also indicate that the fixed point $S=(4.284090909090909$, 4.613636363636363) of Kolmogorov model (3) is also a stable node. Further, if $0<\alpha_{1}<2.5517238126301827$, then fixed point $S=(4.284090909090909,4.613636363636363)$ becomes unstable and meanwhile flip bifurcation occurs, i.e., if $\alpha_{1}=0.967<2.5517238126301827$, then by mathematical computation from (37), one gets $Q_{1}=$ $-0.19445133540974902 \neq 0$. Moreover, from (38), one gets $Q_{2}=0.5853373936670135>0$, which indicates the fact that stable period-2 points bifurcate from $S$, and hence flip bifurcation diagram along with maximum Lyapunov exponents are plotted and presented in Figure 2. Moreover, 3D flip bifurcation diagrams are presented in Figure 3. Finally, Figure 4 shows the complex dynamics with orbits of period$8,9,10,14$.

Hereafter, we will prove the validity of obtained results in Section 5. For instance, if $r_{1}=5.25, r_{2}=5.3, \alpha_{1}=$ 1.98, $\alpha_{2}=0.4, \beta_{1}=2.9, \beta_{2}=2.9$, then from (44)-(46), one gets

$$
\begin{aligned}
& L_{1}:-2.179602154437681-0.005332258840143003 l_{1} \\
& \quad+0.3979708098304923 l_{2}=0,
\end{aligned}
$$

$$
\begin{aligned}
& L_{2}:-0.19000738870159323+0.994667741159857 l_{1} \\
& \quad+0.3979708098304923 l_{2}=0 \\
& \\
& L_{3}:-0.16919692017376872-1.005332258840143 l_{1} \\
& \quad+0.3979708098304923 l_{2}=0 .
\end{aligned}
$$




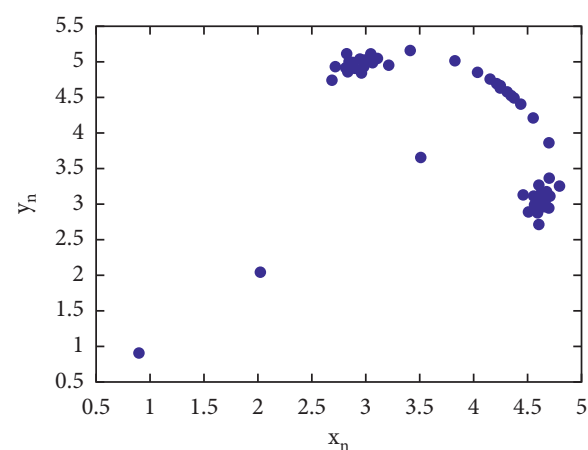

(a)

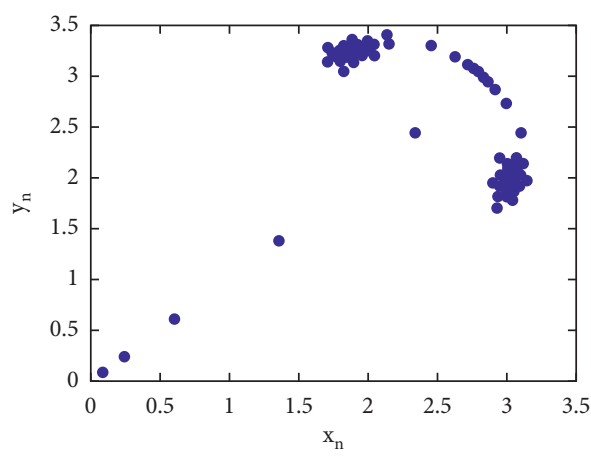

(c)

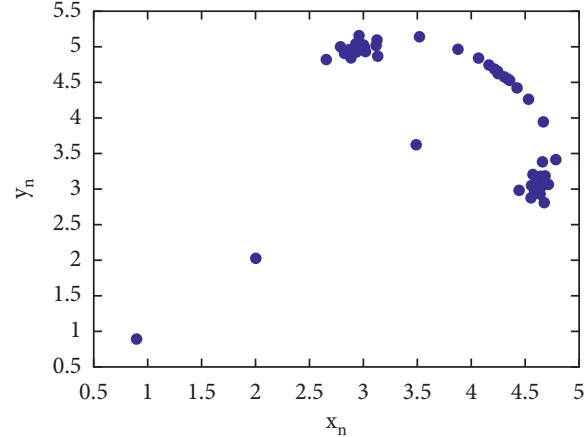

(b)

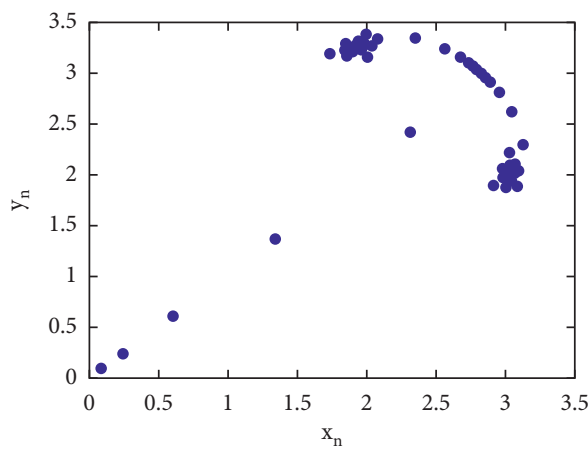

(d)

Figure 1: Stable focus of the Kolmogorov model (3). (a) $\alpha_{1}=2.652$ with $(0.24,0.25)$, (b) $\alpha_{1}=2.6587$ with $(0.294,0.285)$, (c) $\alpha_{1}=2.7$ with $(0.09,0.09)$, and (d) $\alpha_{1}=2.765$ with $(0.092,0.093)$.

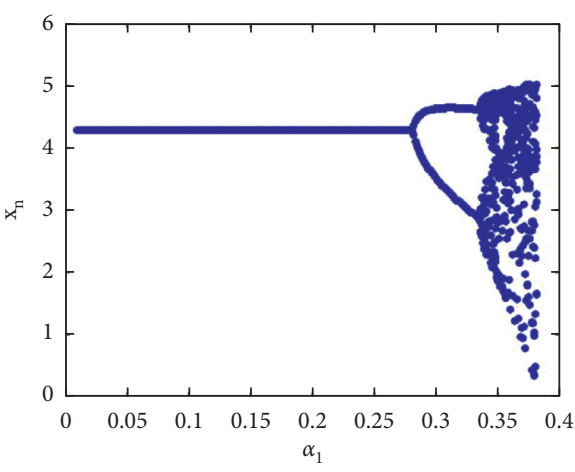

(a)

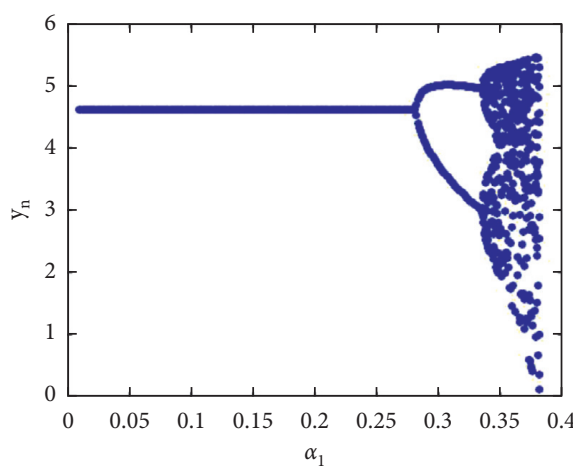

(b)

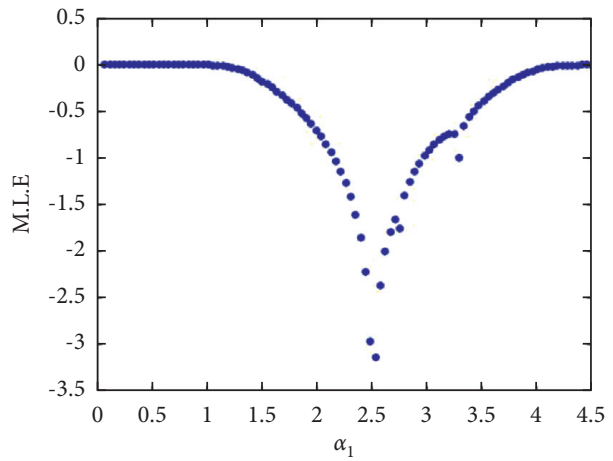

(c)

Figure 2: (a, b) Flip bifurcation diagram of the Kolmogorov model (3) with $\alpha_{1} \in[0.01,2.9]$. (c) Maximum Lyapunov exponents corresponding with $(0.14,0.15)$. 


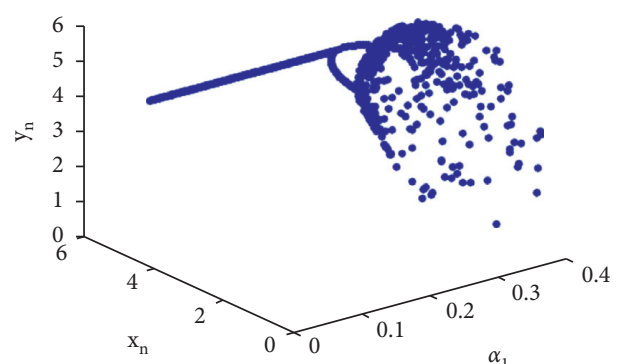

(a)

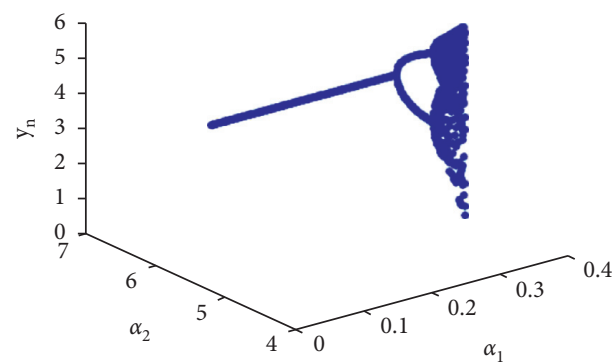

(c)

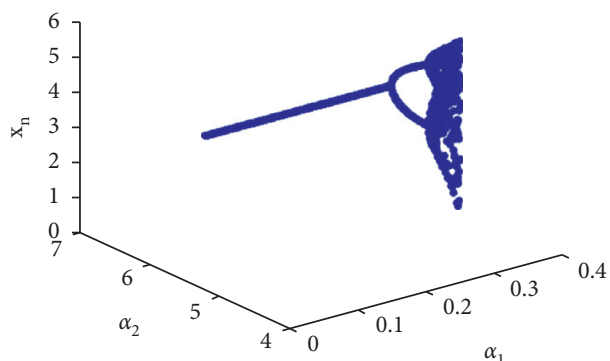

(b)

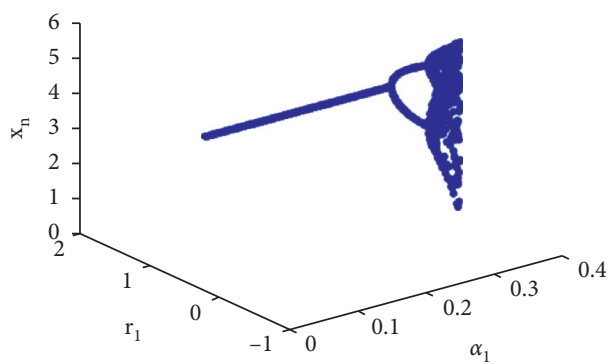

(d)

FIgURE 3: 3D flip bifurcation diagram of the Kolmogorov model (3) with $\alpha_{1} \in[0.01,2.9]$.

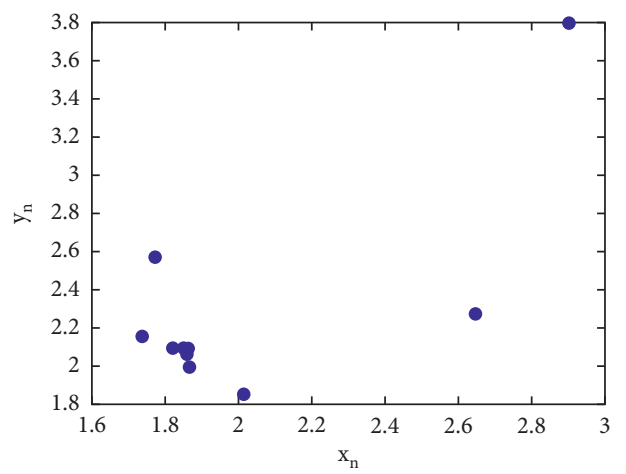

(a)

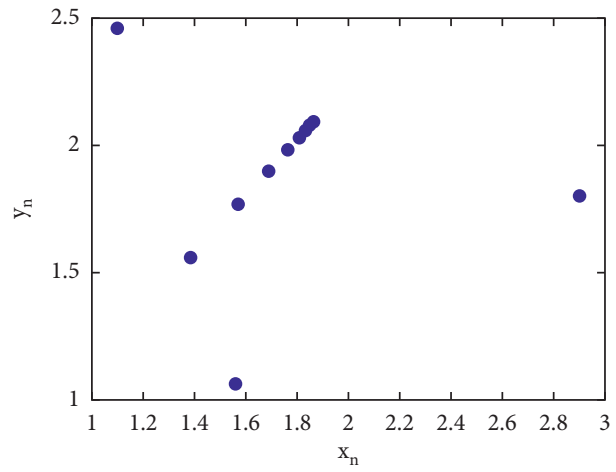

(c)

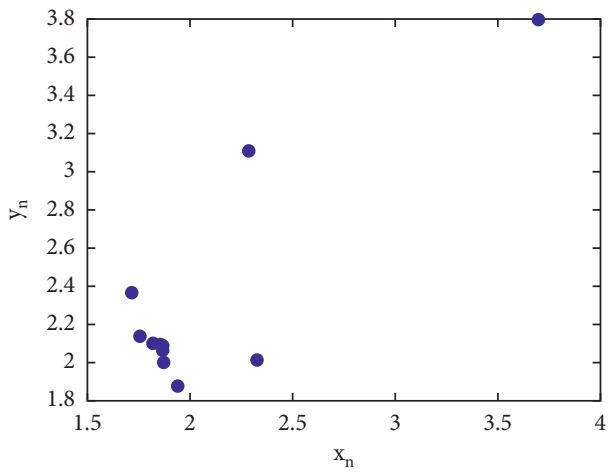

(b)

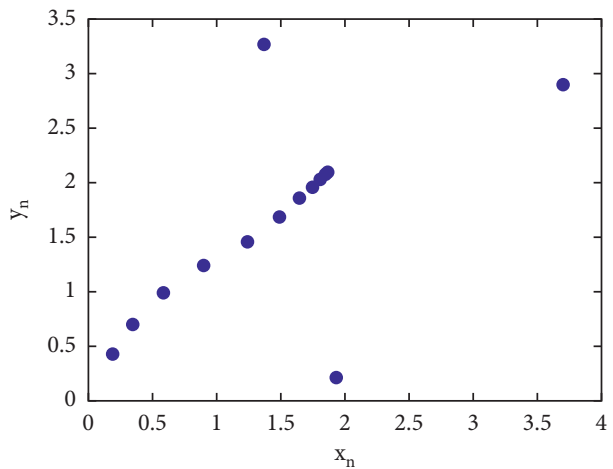

(d)

Figure 4: Complex dynamics of the Kolmogorov model (3). (a) $\alpha_{1}=0.967$ with $(2.9,3.8)$, (b) $\alpha_{1}=1.235$ with $(3.7,3.8)$, (c) $\alpha_{1}=1.7$ with $(2.9,1.8)$, and (d) $\alpha_{1}=1.956$ with $(3.7,3.8)$. 
Hence, lines that are presented in (47)-(49) determine triangular region that gives $\left|\lambda_{1,2}\right|<1$ (see Figure 5).

\section{Boundedness and Global Attractivity of the Kolmogorov Model (3)}

Boundedness with construction of invariant rectangle and global dynamics at interior fixed point of the discrete Kolmogorov model (3) are explored in this section.

7.1. Boundedness Along with Construction of Invariant Rectangle

Theorem 2. Every positive solution $\left\{\left(x_{n}, y_{n}\right)\right\}_{n=0}^{\infty}$ of the Kolmogorov model (3) is bounded if

$$
0<\alpha_{1}<\frac{\left(e^{r_{1}}-1\right)\left(e^{r_{2}}-1\right)}{\alpha_{2} e^{r_{1}+r_{2}}} .
$$

Proof. If $\left\{\left(x_{n}, y_{n}\right)\right\}_{n=0}^{\infty}$ is positive solution of the discrete Kolmogorov model (3), then

$$
\begin{aligned}
& x_{n+1} \leq \frac{e^{r_{1}}}{e^{r_{1}}-1}\left(\beta_{1}+\alpha_{1} y_{n}\right), \\
& y_{n+1} \leq \frac{e^{r_{2}}}{e^{r_{2}}-1}\left(\beta_{2}+\alpha_{2} x_{n}\right) .
\end{aligned}
$$

From $2^{\text {nd }}$ inequality of (51), one gets

$$
y_{n} \leq \frac{e^{r_{2}}}{e^{r_{2}}-1}\left(\beta_{1}+\alpha_{2} x_{n-1}\right) .
$$

Utilizing (52) in the $1^{\text {st }}$ inequality of (51), one gets

$$
x_{n+1} \leq \frac{\beta_{1} e^{r_{1}}}{e^{r_{1}}-1}+\frac{\alpha_{1} \beta_{2} e^{r_{1}+r_{2}}}{\left(e^{r_{1}}-1\right)\left(e^{r_{2}}-1\right)}+\frac{\alpha_{1} \alpha_{2} e^{r_{1}+r_{2}}}{\left(e^{r_{1}}-1\right)\left(e^{r_{2}}-1\right)} x_{n-1} \text {, }
$$

which further gives the following solution:

$$
\begin{aligned}
x_{n+1} \leq & c_{1}\left(\sqrt{\frac{\alpha_{1} \alpha_{2} e^{r_{1}+r_{2}}}{\left(e^{r_{1}}-1\right)\left(e^{r_{2}}-1\right)}}\right)^{n} \\
& +c_{2}\left(-\sqrt{\frac{\alpha_{1} \alpha_{2} e^{r_{1}+r_{2}}}{\left(e^{r_{1}}-1\right)\left(e^{r_{2}}-1\right)}}\right)^{n} \\
& +\frac{\left(\beta_{1} e^{r_{1}} /\left(e^{r_{1}}-1\right)\right)+\left(\alpha_{1} \beta_{2} e^{r_{1}+r_{2}} /\left(\left(e^{r_{1}}-1\right)\left(e^{r_{2}}-1\right)\right)\right)}{1-\left(\alpha_{1} \alpha_{2} e^{r_{1}+r_{2}} /\left(\left(e^{r_{1}}-1\right)\left(e^{r_{2}}-1\right)\right)\right)} .
\end{aligned}
$$

Now, from the $1^{\text {st }}$ inequality of (51), one gets

$$
x_{n} \leq \frac{e^{r_{1}}}{e^{r_{1}}-1}\left(\beta_{1}+\alpha_{1} y_{n-1}\right)
$$

Utilizing (55) in the $2^{\text {nd }}$ inequality of (51), one gets

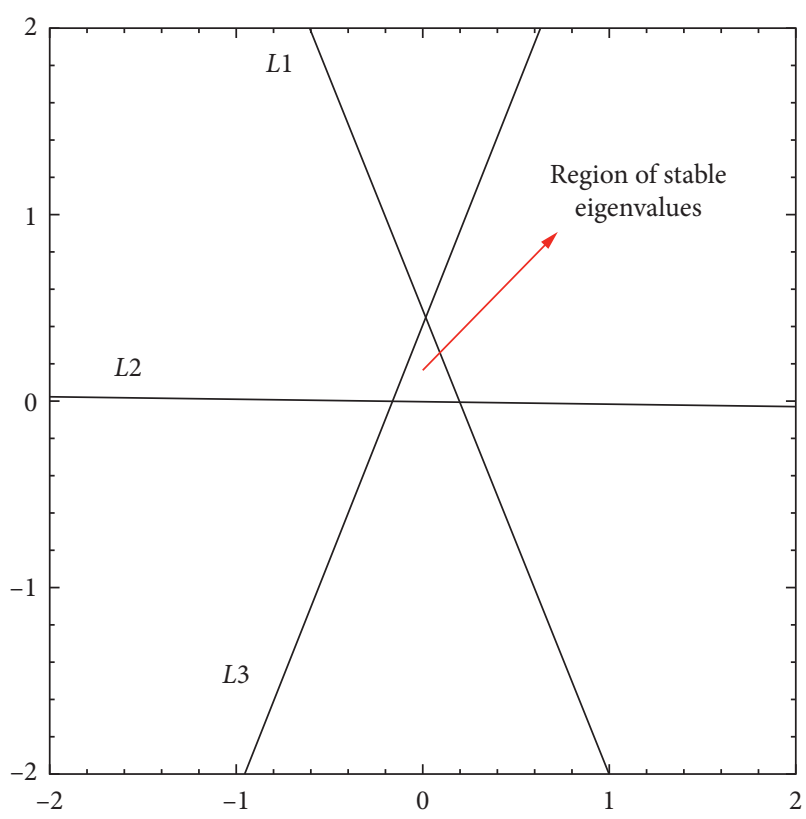

Figure 5: Region of stability where $\left|\lambda_{1,2}\right|<1$.

$y_{n+1} \leq \frac{\beta_{2} e^{r_{2}}}{e^{r_{2}}-1}+\frac{\alpha_{2} \beta_{1} e^{r_{1}+r_{2}}}{\left(e^{r_{1}}-1\right)\left(e^{r_{2}}-1\right)}+\frac{\alpha_{1} \alpha_{2} e^{r_{1}+r_{2}}}{\left(e^{r_{1}}-1\right)\left(e^{r_{2}}-1\right)} y_{n-1}$,

whose solution is

$$
\begin{aligned}
y_{n+1} \leq & c_{3}\left(\sqrt{\frac{\alpha_{1} \alpha_{2} e^{r_{1}+r_{2}}}{\left(e^{r_{1}}-1\right)\left(e^{r_{2}}-1\right)}}\right)^{n} \\
& +c_{4}\left(-\sqrt{\frac{\alpha_{1} \alpha_{2} e^{r_{1}+r_{2}}}{\left(e^{r_{1}}-1\right)\left(e^{r_{2}}-1\right)}}\right)^{n} \\
& +\frac{\left(\beta_{2} e^{r_{2}} /\left(e^{r_{2}}-1\right)\right)+\left(\alpha_{2} \beta_{1} e^{r_{1}+r_{2}} /\left(\left(e^{r_{1}}-1\right)\left(e^{r_{2}}-1\right)\right)\right)}{1-\left(\alpha_{1} \alpha_{2} e^{r_{1}+r_{2}} /\left(\left(e^{r_{1}}-1\right)\left(e^{r_{2}}-1\right)\right)\right)} .
\end{aligned}
$$

If (50) holds, then from (54) and (57), one gets

$$
\begin{aligned}
& 0 \leq x_{n} \leq \frac{\left(\beta_{1} e^{r_{1}} /\left(e^{r_{1}}-1\right)\right)+\left(\alpha_{1} \beta_{2} e^{r_{1}+r_{2}} /\left(\left(e^{r_{1}}-1\right)\left(e^{r_{2}}-1\right)\right)\right)}{1-\left(\alpha_{1} \alpha_{2} e^{r_{1}+r_{2}} /\left(\left(e^{r_{1}}-1\right)\left(e^{r_{2}}-1\right)\right)\right)}, \\
& 0 \leq y_{n} \leq \frac{\left(\beta_{2} e^{r_{2}} /\left(e^{r_{2}}-1\right)\right)+\left(\alpha_{2} \beta_{1} e^{r_{1}+r_{2}} /\left(\left(e^{r_{1}}-1\right)\left(e^{r_{2}}-1\right)\right)\right)}{1-\left(\alpha_{1} \alpha_{2} e^{r_{1}+r_{2}} /\left(\left(e^{r_{1}}-1\right)\left(e^{r_{2}}-1\right)\right)\right)} .
\end{aligned}
$$

Theorem 3. For the discrete Kolmogorov model (3), the invariant rectangle is $\left[0,\left(\left(\left(\beta_{1} e^{r_{1}} /\left(e^{r_{1}}-1\right)\right)+\left(\alpha_{1} \beta_{2} e^{r_{1}+r_{2}} /\right.\right.\right.\right.$ $\left.\left.\left(\left(e^{r_{1}}-1\right)\left(e^{r_{2}}-1\right)\right)\right)\right) /\left(1-\left(\alpha_{1} \alpha_{2} e^{r_{1}+r_{2}} /\left(\left(e^{r_{1}}-1\right) \quad\left(e^{r_{2}}-\right.\right.\right.\right.$ $1)))))] \times\left[0,\left(\left(\left(\beta_{2} e^{r_{2}} /\left(e^{r_{2}}-1\right)\right)+\left(\alpha_{2} \beta_{1} e^{r_{1}+r_{2}} /\left(\left(e^{r_{1}}-1\right)\left(e^{r_{2}}-\right.\right.\right.\right.\right.\right.$ $\left.\left.1)))) /\left(1-\left(\alpha_{1} \alpha_{2} e^{r_{1}+r_{2}} /\left(\left(e^{r_{1}}-1\right)\left(e^{r_{2}}-1\right)\right)\right)\right)\right)\right]$.

Proof. Followed by induction. 
7.2. Attractivity at $S$. From (5), it is noted that $f_{1}$ is increasing in $x \in\left[0,\left(\left(\left(\beta_{1} e^{r_{1}} /\left(e^{r_{1}}-1\right)\right)+\left(\alpha_{1} \beta_{2} e^{r_{1}+r_{2}} /\left(\left(e^{r_{1}}-\right.\right.\right.\right.\right.\right.$ 1) $\left.\left.\left.\left.\left.\left(e^{r_{2}}-1\right)\right)\right)\right) /\left(1-\left(\alpha_{1} \alpha_{2} e^{r_{1}+r_{2}} /\left(\left(e^{r_{1}}-1\right)\left(e^{r_{2}}-1\right)\right)\right)\right)\right)\right]$ (resp . $y \in\left[0,\left(\left(\left(\beta_{2} e^{r_{2}} /\left(e^{r_{2}}-1\right)\right)+\left(\alpha_{2} \beta_{1} e^{r_{1}+r_{2}} /\left(\left(e^{r_{1}}-1\right)\left(e^{r_{2}}-\right.\right.\right.\right.\right.\right.$ $\left.\left.\left.1)))) /\left(1-\left(\alpha_{1} \alpha_{2} e^{r_{1}+r_{2}} /\left(\left(e^{r_{1}}-1\right)\left(e^{r_{2}}-1\right)\right)\right)\right)\right)\right]\right)$ for some fixed $y \in\left[0,\left(\left(\left(\beta_{2} e^{r_{2}} /\left(e^{r_{2}}-1\right)\right)+\left(\alpha_{2} \beta_{1} e^{r_{1}+r_{2}} /\left(\left(e^{r_{1}}-1\right)\left(e^{r_{2}}-\right.\right.\right.\right.\right.\right.$ $\left.\left.1)))) /\left(1-\left(\alpha_{1} \alpha_{2} e^{r_{1}+r_{2}} /\left(\left(e^{r_{1}}-1\right)\left(e^{r_{2}}-1\right)\right)\right)\right)\right)\right] \quad(\operatorname{resp} \cdot x \in[0$, $\left(\left(\left(\beta_{1} e^{r_{1}} /\left(e^{r_{1}}-1\right)\right)+\left(\alpha_{1} \beta_{2} e^{r_{1}+r_{2}} /\left(\left(e^{r_{1}}-1\right) \quad\left(e^{r_{2}}-1\right)\right)\right)\right) /(1-\right.$ $\left.\left.\left.\left.\left(\alpha_{1} \alpha_{2} e^{r_{1}+r_{2}} /\left(\left(e^{r_{1}}-1\right)\left(e^{r_{2}}-1\right)\right)\right)\right)\right)\right]\right)$. Also, $f_{2}$ is increasing in $x \in\left[0,\left(\left(\left(\beta_{1} e^{r_{1}} /\left(e^{r_{1}}-1\right)\right)+\left(\alpha_{1} \beta_{2} e^{r_{1}+r_{2}} /\left(\left(e^{r_{1}}-1\right) \quad\left(e^{r_{2}}-\right.\right.\right.\right.\right.\right.$ $\left.\left.1)))) /\left(1-\left(\alpha_{1} \alpha_{2} e^{r_{1}+r_{2}} /\left(\left(e^{r_{1}}-1\right)\left(e^{r_{2}}-1\right)\right)\right)\right)\right)\right](\operatorname{resp} \cdot y \in[0$, $\left(\left(\left(\beta_{2} e^{r_{2}} / \quad\left(e^{r_{2}}-1\right)\right)+\left(\alpha_{2} \beta_{1} e^{r_{1}+r_{2}} /\left(\left(e^{r_{1}}-1\right)\left(e^{r_{2}}-1\right)\right)\right)\right) /(1-\right.$ $\left.\left.\left.\left.\left(\alpha_{1} \alpha_{2} e^{r_{1}+r_{2}} /\left(\left(e^{r_{1}}-1\right)\left(e^{r_{2}}-1\right)\right)\right)\right)\right)\right]\right)$ for fixed $y \in\left[0,\left(\left(\left(\beta_{2}\right.\right.\right.\right.$ $\left.\left.e^{r_{2}} /\left(e^{r_{2}}-1\right)\right)+\left(\alpha_{2} \beta_{1} e^{r_{1}+r_{2}} /\left(\left(e^{r_{1}}-1\right)\left(e^{r_{2}}-1\right)\right)\right)\right) /\left(1-\left(\alpha_{1} \alpha_{2}\right.\right.$ $\left.\left.\left.\left.e^{r_{1}+r_{2}} /\left(\left(e^{r_{1}}-1\right)\left(e^{r_{2}}-1\right)\right)\right)\right)\right)\right] \quad\left(\operatorname{resp} \cdot x \in\left[0,\left(\left(\left(\beta_{1} e^{r_{1}} /\left(e^{r_{1}}-\right.\right.\right.\right.\right.\right.$ $\left.1))+\left(\alpha_{1} \beta_{2} e^{r_{1}+r_{2}} /\left(\left(e^{r_{1}}-1\right) \quad\left(e^{r_{2}}-1\right)\right)\right)\right) /\left(1-\left(\alpha_{1} \alpha_{2} e^{r_{1}+r_{2}} /\right.\right.$ $\left.\left.\left.\left.\left.\left(\left(e^{r_{1}}-1\right)\left(e^{r_{2}}-1\right)\right)\right)\right)\right)\right]\right)$. This motivates the study of global attractivity at $S$ of discrete Kolmogorov model (3), as follows.

Theorem 4. S of discrete Kolmogorov model (3) is the global attractor.

Proof. Since $f_{1}$ and $f_{2}$ satisfied the above monotonic characteristics and so if $\left(m_{1}, M_{1}, m_{2}, M_{2}\right)$ is the solution of following system:

$$
\begin{aligned}
& m_{1}=f_{1}\left(m_{1}, m_{2}\right), \\
& M_{1}=f_{1}\left(M_{1}, M_{2}\right), \\
& m_{2}=f_{2}\left(m_{1}, m_{2}\right), \\
& M_{2}=f_{2}\left(M_{1}, M_{2}\right) .
\end{aligned}
$$

In view of (3) and (59), one gets

$$
\begin{aligned}
m_{1} & =\frac{e^{r_{1}} m_{1}\left(\beta_{1}+\alpha_{1} m_{2}\right)}{\beta_{1}+\alpha_{1} m_{2}+\left(e^{r_{1}}-1\right) m_{1}}, \\
M_{1} & =\frac{e^{r_{1}} M_{1}\left(\beta_{1}+\alpha_{1} M_{2}\right)}{\beta_{1}+\alpha_{1} M_{2}+\left(e^{r_{1}}-1\right) M_{1}}, \\
m_{2} & =\frac{e^{r_{2}} m_{2}\left(\beta_{2}+\alpha_{2} m_{1}\right)}{\beta_{2}+\alpha_{2} m_{1}+\left(e^{r_{2}}-1\right) m_{2}}, \\
M_{2} & =\frac{e^{r_{2}} M_{2}\left(\beta_{2}+\alpha_{2} M_{1}\right)}{\beta_{2}+\alpha_{2} M_{1}+\left(e^{r_{2}}-1\right) M_{2}} .
\end{aligned}
$$

From (60), one has

$$
\begin{gathered}
\beta_{1}+\alpha_{1} m_{2}+\left(e^{r_{1}}-1\right) m_{1}=e^{r_{1}}\left(\beta_{1}+\alpha_{1} m_{2}\right), \\
\beta_{1}+\alpha_{1} M_{2}+\left(e^{r_{1}}-1\right) M_{1}=e^{r_{1}}\left(\beta_{1}+\alpha_{1} M_{2}\right) .
\end{gathered}
$$

From (61), one has

$$
\begin{aligned}
& \beta_{2}+\alpha_{2} m_{1}+\left(e^{r_{1}}-1\right) m_{2}=e^{r_{2}}\left(\beta_{2}+\alpha_{2} m_{1}\right), \\
& \beta_{2}+\alpha_{2} M_{1}+\left(e^{r_{2}}-1\right) M_{2}=e^{r_{2}}\left(\beta_{2}+\alpha_{2} M_{1}\right) .
\end{aligned}
$$

From (62), one gets

$$
m_{1}-M_{1}=\alpha_{1}\left(m_{2}-M_{2}\right)
$$

Similarly, from (63), one gets

$$
m_{2}-M_{2}=\alpha_{2}\left(m_{1}-M_{1}\right) \text {. }
$$

Form (64) and (65), one gets $\left(\alpha_{1} \alpha_{2}-1\right)\left(m_{1}-M_{1}\right)=0$, which implies that $m_{1}=M_{1}$. Finally, from (65), one gets $m_{2}=M_{2}$. So, $S$ of discrete Kolmogorov model (3) is a global attractor by Theorem 1.16 of [23].

\section{Conclusion and Future Work}

The work is about the local dynamical characteristics at fixed points, existence of periodic points, boundedness, global attractivity, chaos control, and bifurcations of a discrete Kolmogorov model with piecewise-constant argument. By existing linear stability theory, it is proved that fixed point $P$ is never sink, saddle, and nonhyperbolic, but it is a source; $Q$ is never sink, source, and nonhyperbolic, but it is a saddle; $R$ is never sink, source, and nonhyperbolic, but it is a saddle; and finally, $S$ is a stable node if $\alpha_{1}>((1+$ $\left.\left.e^{r_{1}}+e^{r_{2}}+e^{r_{1}+r_{2}}\right) /\left(\alpha_{2}\left(e^{r_{1}}-1\right)\left(e^{r_{2}}-1\right)\right)\right)$, unstable node if $0<\alpha_{1}<\left(\left(1+e^{r_{1}}+e^{r_{2}}+e^{r_{1}+r_{2}}\right) /\left(\alpha_{2}\left(e^{r_{1}}-1\right)\left(e^{r_{2}}-1\right)\right)\right)$, and nonhyperbolic if $\alpha_{1}=\left(\left(1+e^{r_{1}}+e^{r_{2}}+e^{r_{1}+r_{2}}\right) /\left(\alpha_{2}\left(e^{r_{1}}-\right.\right.\right.$ 1) $\left.\left.\left(e^{r_{2}}-1\right)\right)\right)$, but it is never stable focus, unstable focus, and nonhyperbolic. Further, it is proved that fixed points $P, Q, R$, and $S$ are periodic having prime period-1. Then, in order to understand under consideration model deeply, we also explored the occurrence of bifurcations at $S$. It is proved that at $S$, the model undergoes flip bifurcation if $\left.\left(\alpha_{1}, \alpha_{2}, r_{1}, r_{2}, \beta_{1}, \beta_{2}\right) \in \mathscr{F}\right|_{S}$. Further, state feedback control method is utilized in order to stabilize chaos existing in discrete Kolmogorov model (3). Moreover, numerical simulations are also presented to validate obtained results. Finally, boundedness and global attractivity of the Kolmogorov model (3) at $S$ are also explored. The use of robust control to improve the control process and stabilization for the two-species hybrid Kolmogorov model are our next aim to study.

\section{Appendix}

\section{A. Derivation of (3) from (2)}

Dividing the $1^{\text {st }}$ equation of system (2) by $-(x(t))^{2}$ and the $2^{\text {nd }}$ equation of system (2) by $-(y(t))^{2}$, one gets

$$
\begin{aligned}
& -\frac{1}{(x(t))^{2}} \frac{\mathrm{d} x(t)}{\mathrm{d} t}+\frac{r_{1}}{x(t)}=\frac{r_{1}}{\beta_{1}+\alpha_{1} y(n)}, \\
& -\frac{1}{(y(t))^{2}} \frac{\mathrm{d} y(t)}{\mathrm{d} t}+\frac{r_{2}}{y(t)}=\frac{r_{2}}{\beta_{2}+\alpha_{2} x(n)} .
\end{aligned}
$$

Now, putting $(1 / x(t))=v$ in the $1^{\text {st }}$ equation of system (A.1) and $(1 / y(t))=z$ in the $2^{\text {nd }}$ equation of system (A.1), one gets

$$
\begin{aligned}
& \frac{\mathrm{d} v}{\mathrm{~d} t}+r_{1} v=\frac{r_{1}}{\beta_{1}+\alpha_{1} y(n)}, \\
& \frac{\mathrm{d} z}{\mathrm{~d} t}+r_{2} z=\frac{r_{2}}{\beta_{2}+\alpha_{2} x(n)} .
\end{aligned}
$$


Moreover, multiplying the $1^{\text {st }}$ equation of system (A.2) by integrating factor $e^{r_{1} t}$ and the $2^{\text {nd }}$ equation of system (A.2) by integrating factor $e^{r_{2} t}$, one gets

$$
\begin{aligned}
& \mathrm{d}\left(\frac{1}{x(t)} e^{r_{1} t}\right)=\frac{r_{1} e^{r_{1} t}}{\beta_{1}+\alpha_{1} y(n)}, \\
& \mathrm{d}\left(\frac{1}{y(t)} e^{r_{2} t}\right)=\frac{r_{2} e^{r_{2} t}}{\beta_{2}+\alpha_{2} x(n)} .
\end{aligned}
$$

Now, integrating from $n \longrightarrow t$ and $t \longrightarrow n+1$, one gets

$$
\begin{aligned}
& \int_{n}^{t} \mathrm{~d}\left(\frac{1}{x(t)} e^{r_{1} t}\right)=\frac{r_{1}}{\beta_{1}+\alpha_{1} y(n)} \int_{n}^{t} e^{r_{1} t} \mathrm{~d} t \\
& \int_{n}^{t} \mathrm{~d}\left(\frac{1}{y(t)} e^{r_{2} t}\right)=\frac{r_{2}}{\beta_{2}+\alpha_{2} x(n)} \int_{n}^{t} e^{r_{2} t} \mathrm{~d} t
\end{aligned}
$$

After some simplifications, the solution of (A.4) becomes

$$
\begin{aligned}
& x_{n+1}=\frac{e^{r_{1}} x_{n}\left(\beta_{1}+\alpha_{1} y_{n}\right)}{\beta_{1}+\alpha_{1} y_{n}+\left(e^{r_{1}}-1\right) x_{n}}, \\
& y_{n+1}=\frac{e^{r_{2}} y_{n}\left(\beta_{2}+\alpha_{2} x_{n}\right)}{\beta_{2}+\alpha_{2} x_{n}+\left(e^{r_{2}}-1\right) y_{n}},
\end{aligned}
$$

which can also be written as

$$
\begin{aligned}
& x_{n+1}=\frac{e^{r_{1}} x_{n}}{1+\left(\left(e^{r_{1}}-1\right) /\left(\beta_{1}+\alpha_{1} y_{n}\right)\right) x_{n}}, \\
& y_{n+1}=\frac{e^{r_{2}} y_{n}}{1+\left(\left(e^{r_{2}}-1\right) /\left(\beta_{2}+\alpha_{2} x_{n}\right)\right) y_{n}} .
\end{aligned}
$$

\section{Data Availability}

All the data utilized in this article have been included, and the sources from where they were adopted were cited accordingly.

\section{Conflicts of Interest}

The author declares no conflicts of interest regarding the publication of this paper.

\section{References}

[1] K. Yang, X. Xie, and F. Chen, "Global stability of a discrete mutualism model," Abstract and Applied Analysis, vol. 2014, Article ID 709124, 7 pages, 2014.

[2] R. May, Theoretical Ecology: Principles and Applications, R. M. May, Ed., Oxford University PressSalt Lake City, UT, USA, 1976.

[3] S. Bhattacharya and L. M. Saha, "A model of discrete Kolmogorov-type competitive interaction in a two-species ecosystem," arXiv:1507.07645, 2015.

[4] L. Cheng and H. Cao, "Bifurcation analysis of a discrete-time ratio-dependent predator-prey model with Allee effect," Communications in Nonlinear Science and Numerical Simulation, vol. 38, pp. 288-302, 2016.
[5] Z. Jing and J. Yang, "Bifurcation and chaos in discrete-time predator-prey system," Chaos, Solitons \& Fractals, vol. 27, no. 1, pp. 259-277, 2006.

[6] F. I. G. E. N. Kangalgil and N. I. L. U. F. E. R. Topsakal, "Stability analysis and flip bifurcation of a discrete-time preypredator model with predator immigration," Asian Journal of Mathematics and Computer Research, vol. 27, no. 3, pp. 1-10, 2020.

[7] J. Ran, Y.-Q. Li, S.-J. Ma, and J. Wu, "Hopf bifurcation analysis for a stochastic discrete-time hyperchaotic system," Discrete Dynamics in Nature and Society, vol. 2015, Article ID 127404, 12 pages, 2015.

[8] J. R. Beddington, C. A. Free, and J. H. Lawton, "Dynamic complexity in predator-prey models framed in difference equations," Nature, vol. 255, no. 5503, pp. 58-60, 1975.

[9] F. Chen, "Permanence and global attractivity of a discrete multispecies Lotka-Volterra competition predator-prey systems," Applied Mathematics and Computation, vol. 182, no. 1, pp. 3-12, 2006.

[10] C. Lu and L. Zhang, "Permanence and global attractivity of a discrete semi-ratio dependent predator-prey system with holling ii type functional response," Journal of Applied Mathematics and Computing, vol. 33, no. 1-2, pp. 125-135, 2010.

[11] N. Fang and X. X. Chen, "Permanence of a discrete multispecies lotka-volterra competition predator-prey system with delays," Nonlinear Analysis: Real World Applications, vol. 9, no. 5, pp. 2185-2195, 2008.

[12] Q. Fang, X. Li, and M. Cao, "Dynamics of a discrete predatorprey system with Beddington-Deangelis function response," Applied Mathematics, vol. 3, no. 4, pp. 389-394, 2012.

[13] D. Jana and G. P. Samanta, "Significance of prey harvesting in prey-predator system in discrete time scale using interval parameters," International Journal of Ecological Economics \& Statistics, vol. 39, no. 2, pp. 46-60, 2018.

[14] J. Guckenheimer and P. Holmes, Nonlinear Oscillations, Dynamical Systems and Bifurcation of Vector Fields, SpringerVerlag, New York, NY, USA, 1983.

[15] Y. A. Kuznetsov, Elements of Applied Bifurcation Theorey, Springer-Verlag, New York, NY, USA, 3rd edition, 2004.

[16] E.-G. Gu, "The nonlinear analysis on a discrete host-parasitoid model with pesticidal interference," Communications in Nonlinear Science and Numerical Simulation, vol. 14, no. 6, pp. 2720-2727, 2009.

[17] H. N. Agiza, E. M. ELabbasy, H. EL-Metwally, and A. A. Elsadany, "Chaotic dynamics of a discrete prey-predator model with holling type II," Nonlinear Analysis: Real World Applications, vol. 10, no. 1, pp. 116-129, 2009.

[18] H.-F. Huo and W.-T. Li, "Stable periodic solution of the discrete periodic Leslie-gower predator-prey model," Mathematical and Computer Modelling, vol. 40, no. 3-4, pp. 261269, 2004.

[19] L. Li and Z.-J. Wang, "Global stability of periodic solutions for a discrete predator-prey system with functional response," Nonlinear Dynamics, vol. 72, pp. 507-516, 2013.

[20] X. Chen, "Periodicity in a nonlinear discrete predator-prey system with state dependent delays," Nonlinear Analysis: Real World Applications, vol. 8, no. 2, pp. 435-446, 2007.

[21] S. N. Elaydi, An Introduction to Difference Equations, Springer-Verlag, New York, NY, USA, 1996.

[22] S. Lynch, Dynamical Systems with Applications Using Mathematica, Birkhäuser, Boston, MA, USA, 2007.

[23] E. A. Grove and G. Ladas, Periodicities in Nonlinear Difference Equations, Vol. 4, CRC Press, Boca Raton, FL, USA, 2004. 